\title{
DETC2005-84241
}

\section{AN APPROACH FOR COMPENSATION OF GEOMETRIC FAULTS IN MACHINE TOOLS}

\author{
Christian Rudolf* \\ Jörg Wauer \\ Institut für Technische Mechanik \\ Universität Karlsruhe (TH) \\ Kaiserstr. 12 \\ 76131 Karlsruhe, Germany \\ Email: rudolf@itm.uni-karlsruhe.de
}

\author{
Christian Munzinger \\ Jürgen Fleischer \\ Institut für Produktionstechnik \\ Universität Karlsruhe (TH) \\ Kaiserstr. 12 \\ 76131 Karlsruhe, Germany
}

\begin{abstract}
Geometric faults in parts of machine tools with parallel kinematics lead to stresses in the structure and deflections of the tool center point, reducing the quality of the workpiece. Improving the design of machine tools can reduce these influences. In this paper an approach to compensate the influence of geometric faults in parallel kinematics based on the design of an adaptronic strut is introduced. The strut is divided in two halves and two piezoelectric transducers are implemented in between them, used as sensor and actuator, respectively. A preliminary design of the adaptronic strut is presented. The problems of measuring low-frequency signals using piezoelectric transducers are considered in the design.

Finally, a primary analytical model of the dynamical behavior of the adaptronic compensation unit is presented. The strut and its connection to the surroundings are regarded as a flexible multibody system, the equations of motion are derived using linear graph theory. Some simulation results are presented.
\end{abstract}

Keywords: parallel kinematics, compensation of geometric faults, piezoelectric transducers, linear graph theory.

\footnotetext{
*Address all correspondence to this author.
}

\section{INTRODUCTION}

In machine tools, static, quasi-static, thermic, and dynamic influences result in deflections of the parts and, most important, of the tool center point (TCP). Hocken [1] defines quasi-static faults to be relative faults of position between the tool and the workpiece of relatively large time constants, depending on the structure of the machine tool. They usually result from geometric faults of the lines of the guiding skids, static loads such as gravity influences or static process loads. In machine tools with parallel kinematics, there are additional influences like production tolerances, installation faults or thermic deformations causing quasi-static faults.

Furthermore, vibrations during the production process and changes of temperature within the assemblies of the machine tool result in dynamic and thermal faults, respectively.

All these faults lead to stresses within the structure of the machine tool and in time variant deflections of the TCP. Thus, the quality of the production process and therefore the quality of the workpiece is reduced.

According to Stengele [2], most concepts of machine tools only include three translational axes and an additional rotatory axis for machining. This suggests, that most future parallel kinematic machine tools will be conceived with up to three degrees of freedom (DOF). Within such parallel kinematic machine tools with two or three degrees of freedom, the rotational degrees of freedom are kinematically locked, as shown exemplarily in Fig. (1). 


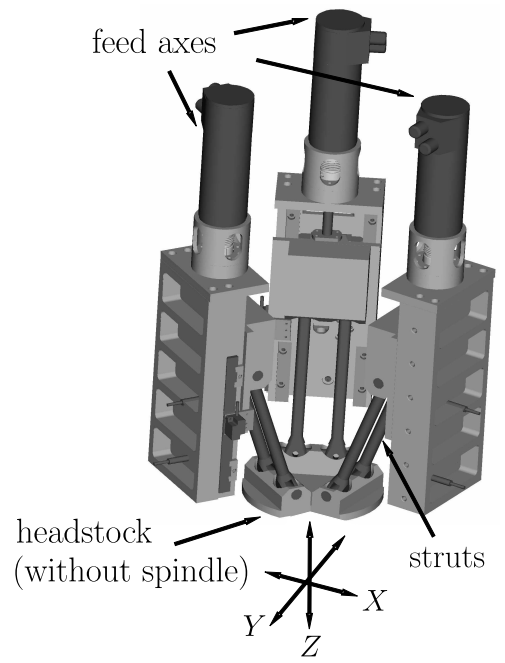

Figure 1. PARALLEL KINEMATIC MACHINE TOOL WITH THREE TRANSLATIONAL DEGREES OF FREEDOM.

Then, the quasi-static geometric faults mentioned above and the faults due to installation result in stresses within the whole structure. Thus, a machine tool of ideally two or three degrees of freedom can exhibit a full three-dimensional kinematic behavior in six axes.

The resulting deflections can be divided in rotational and translational faults of the machine tool. The translational faults usually can be compensated using the machine axes of the guided skids, whereas the compensation of the rotational faults using the same axes is rather limited. For the turning process, for example, there is point contact between the tool and the workpiece. A small angular deflection can be corrected or it might even be negligible. However, there is line contact between tool and workpiece for e.g. boring or milling processes, and an angular deflection of the TCP directly results in shape faults of the workpiece.

Thus, for machine tools with parallel kinematics of two or three translational degrees of freedom, geometric faults within the structure have to be compensated. Using piezoelectric transducers as actuators, the approaches for compensation of geometric faults and thermal deflections within machine tools presented in [3-6] are promising.

This paper seizes these procedures and introduces an adaptronic strut. The strut includes two piezoelectric transducers, one of them is used as sensor element to measure the stresses in the strut in-situ, the other one is used as actuator element. To overcome the problems with measuring low-frequency signals using piezoelectric sensors $[7,8]$, the concept of a balance with a vibrating string is adapted.

Finally, a model of the adaptronic strut and its connection to the surroundings is presented. Considering the strut as a multi-body system including flexible elements, the equations of motion of the system are derived and some simulation results are presented.

\section{ADAPTRONIC STRUT}

For a machine tool with parallel kinematics as shown in Fig. (1) an adaptronic strut was designed. The strut has to fulfill the following requirements: It must have the ability

- to compensate deflections resulting from process loads. This results in a strut variable in length to compensate axial deviations due to changes in the process load.

- to compensate deflections resulting from geometric faults in the structure.

The length of the strut is controlled by a pre-calculated state of stress within the strut.

- and to maintain the bending stiffness and torsional stiffness of the original strut to the greatest possible extent.

The first and the second requirement use the same input and same output variables. Thus, both can be fulfilled by just one compensation unit. A piezoelectric transducer is a well-suited element for that and it can be used for both, actuating the necessary travels and measuring the current axial stress in the strut. According to Ruschmeyer [7], by using piezoelectric sensors it is cumbersome and difficult to measure low-frequency signals, and it is impossible to measure static signals. As geometric faults and quasistatic process loads result in static signals, an additional concept to measure these signals with the piezoelectric transducer was implemented in the design of the strut.

\section{Balance With A Vibrating String}

To transform static or low-frequency loads in dynamic or higher-frequency loads, which are measurable with piezoelectric sensors, the concept of a balance with a vibrating string was adopted.

A transversally vibrating string induces an axial load on the bearings at both ends. This load can be transferred onto the piezoelectric transducer in the strut by a suited design of the strut. The signal measured by the sensor is a direct current voltage, due to the constant state-of-stress induced by deflections and / or quasi-static loads, and an alternating current voltage, due to the vibration of the string, as exemplified in Fig. (2). Using a low-pass filter or a high-pass filter, the interesting part of the signal can easily be extracted from the sensor signal. Adopting this concept, the required pre-stress for the use of piezoelectric transducers additionally was achieved.

\section{Design}

The adaptronic strut was designed as shown in Fig. (3). The strut basically consists of a lower and an upper half. In between these halves, a sensor-actuator unit is implemented. For primary simplicity, sensor function and actuator function are split and two 


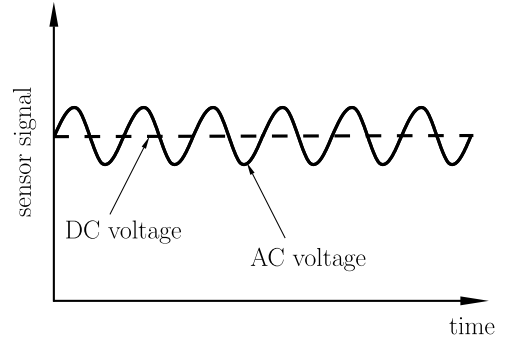

Figure 2. EXEMPLIFIED SENSOR SIGNAL.
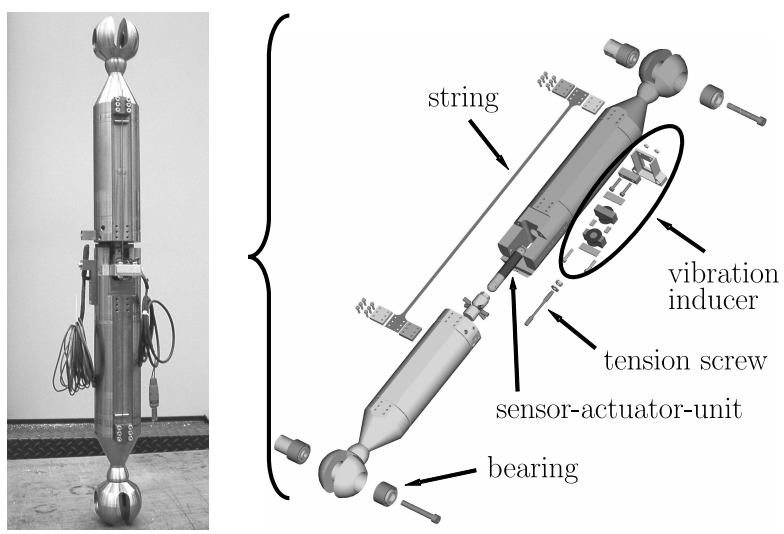

Figure 3. ADAPTRONIC STRUT.

piezoelectric stack transducers were built-in. The halves of the strut are connected by a knife-edge bearing with line-contact and a tension screw, which is preventing the separation of the two halves. Along one side of the strut there are clamps to mount the string. The position of the clamps is offset to the center of the longitudinal axis of the strut. This offset lever increases the sensor signal induced by the vibrating string. The positions of the clamps along the strut are variable and so, the length of the string can be varied. That offers the possibility to set the eigenfrequency $f_{0}$ of the string according

$$
f_{0}=\frac{1}{2 l_{S}} \sqrt{\frac{T}{A \rho}},
$$

where $T$ is the longitudinal force on the string and $l_{S}, A$ and $\rho$ are its length, cross-sectional area and density, respectively. On the same side of the strut an electro-magnetic device to induce artificial vibrations of the string is mounted. This device is preliminary implemented in the design of the strut but it will be dismantled later on, since the vibrations during the machining process will be sufficient to start the vibration of the string. The shape of the developed adaptronic strut, e.g. the overall length
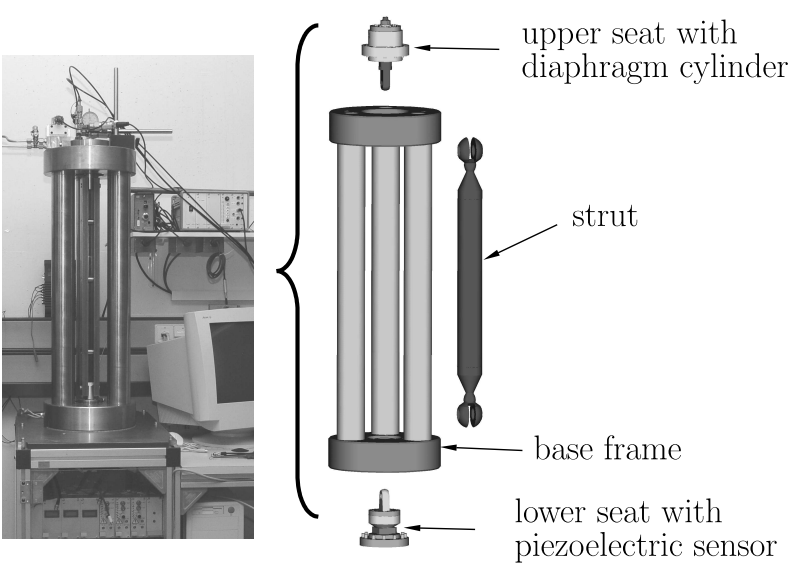

Figure 4. EXPERIMENTAL SET-UP.

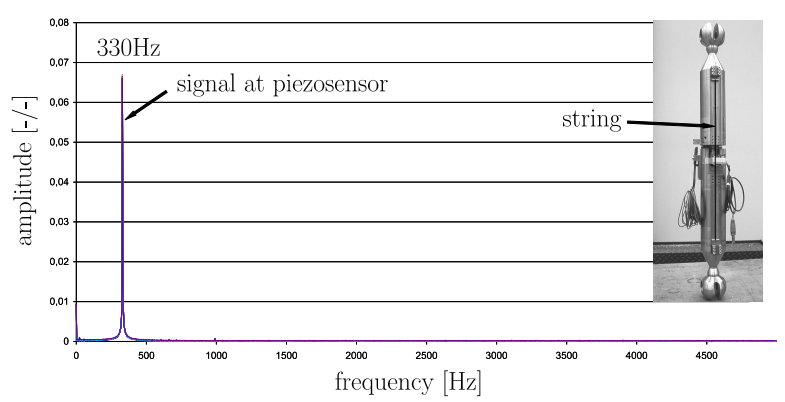

Figure 5. SIGNAL AT THE PIEZOELECTRIC SENSOR.

and the shape of the bearings at the ends, is similar to the design of struts used in various concepts of machine tools with parallel kinematics [9].

For preliminary analysis an experimental test rig was built, which is shown in Fig. (4). The test rig consists of a base frame including two seats for the strut. In the lower seat a piezoelectric sensor is implemented to measure the longitudinal stresses of the strut during the experiment, independent of the integrated sensor element. In the upper seat a membrane cylinder is built-in to apply simulated process loads. The strut was placed in the test rig and a string $\left(d=1 \mathrm{~mm}, l_{S}=0.6 \mathrm{~m}\right)$ was clamped on its side. The string, which was under pre-stress $T \approx 800 \mathrm{~N}$, was excited with a frequency of $f=330 \mathrm{~Hz}$. The experiment was repeated 10 times and the sensor signal was examined. Figure (5) shows the Fourier analysis of all sensor signals. The measured amplitudes of the voltage at the piezo sensor ranged between $0.5-2.0 \mathrm{~V}$ and were measurable without any expensive amplification.

Due to the splitting of the strut to implement the piezoelectric transducers the torsional stiffness and the bending stiffness of the adaptronic strut were reduced. The combination of the bearing point and the piezoelectric actuator can increase the 


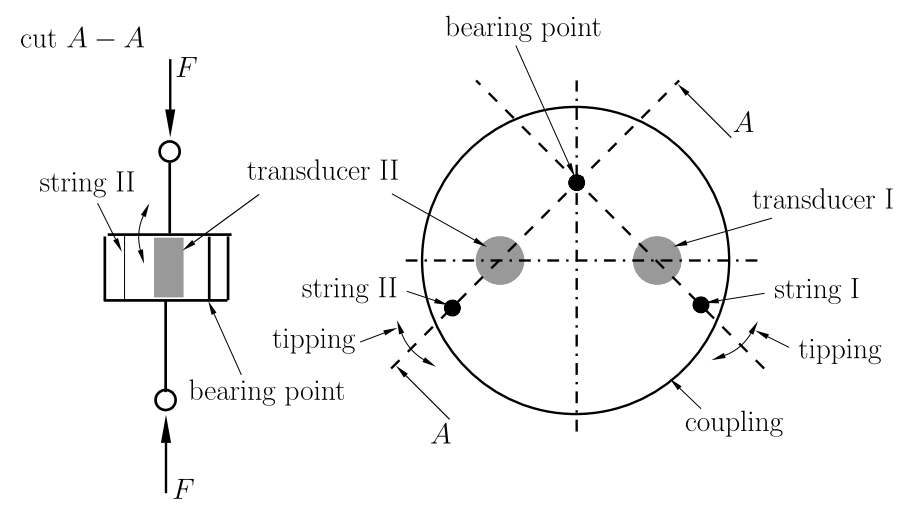

Figure 6. STRUT DESIGN.

bending stiffness about the axis perpendicular to the line through these two points but not about the line itself. A possible solution for this problem might be the implementation of a second sensor-actuator unit placed as shown in Fig. (6). A tipping due to the travel of piezo-actuator $I$ is compensated by actuator $I I$ and vice versa.

The torsional stiffness is increased by the installation of a metal bellow coupling around the cut. The coupling additionally seals the adaptronic compensation unit against the surroundings.

\section{DERIVATION OF THE EQUATIONS OF MOTION}

The examined system comprises the adaptronic strut, consisting of an upper and a lower half and two piezoelectric transducers in between. The upper part of the strut, which is inclined about an angle $\psi$ against the vertical axis, is connected to a horizontal axle by a rotational joint, whose rotation axis is perpendicular to the longitudinal axis of the axle. The axle can rotate about its longitudinal axis, connected by this rotational joint to a vertically guided skid. The skid connects the whole system to the surroundings.

The skid and the axle are considered as rigid bodies, the two halves of the strut as well as the two piezoelectric transducers are modeled as flexible bodies. The four flexible bodies are primarily modeled to be rigidly coupled. All joints in the system are ideal.

The equations of motion of the system are generated using DynaFlex, a symbolic package for multi-body dynamics $[10,11]$. However, DynaFlex doesn't have the ability to model the electric behavior of piezoelectric materials. Thus, the electrical properties have to be implemented artificially.

\section{Modeling Of The Piezoelectric Transducer}

According to $[12,13]$, the linear constitutive equations of a piezoelectric transducer read

$$
\begin{aligned}
& T_{p}=c_{p q}^{E} S_{q}-e_{k p} E_{k}, \\
& D_{i}=e_{i q} S_{q}+\varepsilon_{i k}^{S} E_{k},
\end{aligned}
$$

where $T_{p}$ and $S_{q}$ are the stress and strain in the piezoelectric material, $E_{k}$ is the electric field and $D_{i}$ is the electric displacement. The piezoelectric constants are $c_{p q}^{E}, e_{k p}=e_{i q}$ and $\varepsilon_{i k}^{S}$. For the designed adaptronic strut only the dynamic behavior of the piezoelectric transducer in its axial direction (3-direction) influences the system. Since stack transducers with $n$ equally sized plates are used and the relation between the plate thickness $h$ and the plate diameter $d$ is $h \ll d$, a homogeneous electric field in axial direction is assumed and electric leakage fields in transversal directions are negligible. Furthermore, normal stresses in transversal directions, shear stresses and shear strains are neglected. Thus, Eqn. (2) and (3) reduce to

$$
\begin{aligned}
T_{3} & =\alpha S_{3}-\beta E_{3}, \\
D_{3} & =\beta S_{3}+\gamma E_{3},
\end{aligned}
$$

where $\alpha, \beta$ and $\gamma$ are abbreviations for the following combinations of the piezoelectric constants:

$$
\begin{aligned}
& \alpha=\frac{1}{s_{33}^{E}}=\left(c_{33}^{E}-\frac{2\left(c_{13}^{E}\right)^{2}}{c_{11}^{E}+c_{12}^{E}}\right), \\
& \beta=\frac{d_{33}}{s_{33}}=\left(e_{33}-\frac{2 c_{13}^{E} e_{31}}{c_{11}^{E}+c_{12}^{E}}\right), \\
& \gamma=\varepsilon_{33}^{T}-\frac{d_{33}^{2}}{s_{33}^{E}}=\left(\varepsilon_{33}^{S}-\frac{2\left(e_{31}\right)^{2}}{c_{11}^{E}+c_{12}^{E}}\right) .
\end{aligned}
$$

Using Eqn. (4), the electric force component of the piezoelectric actuator can be determined directly. The mechanical and electric relations for axial stress $T_{3}$, axial strain $S_{3}$ and homogeneous electric field $E_{3}$ are

$$
T_{3}=\frac{F}{A}, S_{3}=\frac{\Delta l}{l}, E_{3}=n \frac{U}{l}
$$

Substituting these expressions in Eqn. (4) yields

$$
F=\underbrace{\alpha \frac{A}{l} \Delta l}_{F_{\text {mech }}}-\underbrace{n \beta \frac{A}{l} U}_{F_{e l}} .
$$


The actuator force consists of a mechanical component due to the displacement $\Delta l$ and an electric component due to the voltage $U$. The mechanical part, however, including the inertia effects of the material, is already implemented in the multi-body system model with the piezoelectric transducer considered as a flexible body.The electrical part of the transducer force has to be included as an additional force, depending on the input voltage $U$.

To describe the electric behavior of the piezoelectric sensor, additionally Eqn. (5) has to be taken into account. The sensor is assumed to be ideal, i.e. the electrodes are ideally isolated and leakage currents due to the limited electrical resistance of the transducer are neglected. The charge density $\sigma$ and therefore the electric displacement $D_{3}$ are zero $[14,15]$, i.e.

$$
\sigma=D_{3}=\frac{Q}{A}=0
$$

and Eqn. (5) can be solved for

$$
U=-\frac{1}{n} \frac{\beta}{\gamma} \Delta l
$$

such that Eqn. (10) finally reads

$$
F=\underbrace{\alpha \frac{A}{l} \Delta l}_{F_{\text {mech }}}+\underbrace{\frac{A}{l} \frac{\beta^{2}}{\gamma} \Delta l}_{F_{e l}} .
$$

Introducing a mechanical spring stiffness $c_{\text {mech }}$ and an electrical spring stiffness $c_{e l}$, Eqn. (13) can be rewritten as

$$
F=c_{m e c h} \Delta l+c_{e l} \Delta l
$$

and the transducer force can be interpreted as a system of two parallel springs. Again, the mechanical part of the piezoelectric element is already implemented in the model by a flexible body. Thus, only the electrical force component of the sensor in Eqn. (13) is artificially implemented either as a spring with spring constant $c=c_{e l}$ or by introducing the additional force $F_{e l}$, depending on the elongation $\Delta l$.

\section{Graph-Theoretic Modeling}

DynaFlex needs as input the description of the system as a linear graph. A linear graph consists of nodes (dots) and edges (lines) and often it is used to describe the structure of a multibody system. The nodes usually represent the bodies of the system and application points of joints and forces on the bodies, the edges connect all points with each other, representing joints as

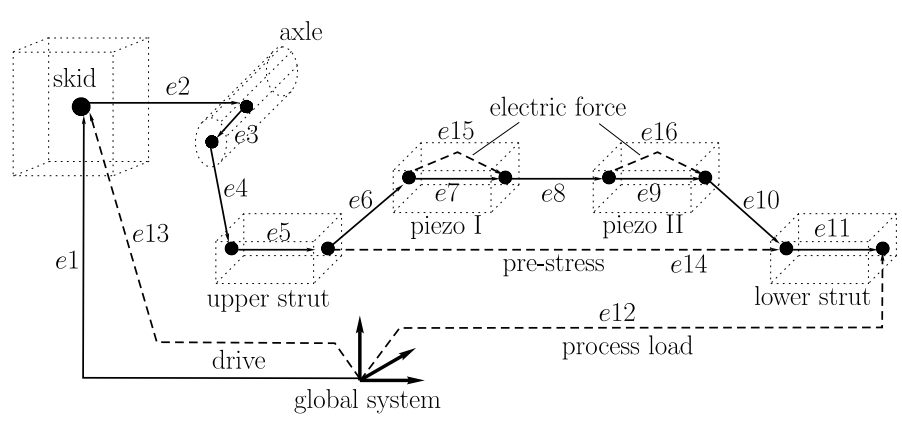

Figure 7. LINEAR GRAPH.

well as force or motion drivers. For further information see [16]. The linear graph representation of the system of the strut and its connection to the surroundings is shown in Fig. (7). The solid edges $e 1, e 2$ and $e 4$ describe the vertical translational joint of the skid and the two rotational joints of the axle and the strut, respectively. The fixed joints, connecting the flexible bodies with each other, are represented by edges $e 6, e 8$ and $e 10$. Edge $e 3$ describes the location of the rotational joint that connects the axle and the upper half of the strut. Edges $e 5, e 7, e 9$ and $e 11$ on the one hand represent the position of the points where the flexible bodies are connected with each other, and on the other hand they describe the flexible behavior of the two parts of the strut and of the two piezoelectric transducers.

The dashed edges represent external forces acting on the system, such as the process loads $(e 12)$ or the driving force of the skid (e13). Furthermore, they describe the electric components of the forces of the piezoelectric transducers $(e 15, e 16)$ and the pre-stress applied onto the transducers $(e 14)$. Other edges representing, for example, gravity influences or centers of mass of the bodies are not shown for clarity.

DynaFlex generates the equations of motion in the form

$$
\mathbf{M}(\underset{\sim}{q}) \cdot \underset{\sim}{\ddot{q}}=\mathbf{F}(\underset{\sim}{q}, \underset{\sim}{\dot{q}}, t)
$$

with mass matrix $\mathbf{M}$ and force vector $\mathbf{F}$. The system has several degrees of freedom, described by the components $q_{i}$ of the coordinate vector $\underset{\sim}{q}$. Three degrees of freedom are due to the motion along the translational joint of the guidance of the skid and the two rotations of the axle and the strut. Furthermore, there are several coordinates describing the behavior of the flexible bodies. The flexible bodies are modeled as Euler-Bernoulli beams, discretized using the Rayleigh-Ritz method. The describing coordinates are the axial deformation $u$, the bending deformations $v$ and $w$ and the torsion $\phi$ of the beam [10].

The linear graph of the system remains the same independent of the number of coordinates describing the flexible bodies. Thus, the flexible behavior of the strut and the piezoelectric transducers can be refined easily. 
Table 1. TRANSDUCER PARAMETERS.

\begin{tabular}{cccc}
\hline Parameter & Unit & Actuator & Sensor \\
\hline$l$ & $\mathrm{~m}$ & 0.046 & 0.016 \\
$d$ & $\mathrm{~m}$ & 0.015 & 0.015 \\
$\rho$ & $\mathrm{kg} / \mathrm{m}^{3}$ & 7670 & 7670 \\
$\varepsilon_{33}^{T} / \varepsilon_{0}$ & - & 950 & 950 \\
$d_{33}$ & $\mathrm{C} / \mathrm{N}(\mathrm{m} / \mathrm{V})$ & $425 \cdot 10^{-12}$ & $425 \cdot 10^{-12}$ \\
$s_{33}^{E}$ & $\mathrm{~m}^{2} / \mathrm{N}$ & $27.3 \cdot 10^{-12}$ & $27.3 \cdot 10^{-12}$ \\
\hline
\end{tabular}

\section{SIMULATION RESULTS}

The piezoelectric transducers used in the adaptronic strut designed are described by the parameters listed in Table 1 .

During the first simulations the model was reduced to a strut which was not able to move. The translational joint and both rotational joints were fixed. As in the test rig, the strut was vertically positioned with $\psi=180^{\circ}$, i.e. the lower part of the strut was on top. The strut was primarily examined in its axial behavior only. Thus, all degrees of freedom describing the torsion or the bending of the transducers and the two halves of the strut were not considered. The longitudinal behavior was described by two axial coordinates for each flexible body, modeling the elongation of the bodies with a quadratic function. Thus, the system was described by a total of eight deformation variables.

To validate the symbolic 8-DOF model of the adaptronic strut, a similar model of the strut was built in the proven commercial software package ADAMS and the simulation results of both models were compared with each other.

\section{Piezoelectric Sensor}

In a first step, the behavior of the piezoelectric sensor element within the adaptronic strut was simulatively examined. At the top, the strut was loaded by a cycling load of frequency $f=330 \mathrm{~Hz}$. Since the strut was vertically placed, the point of load incidence represents the position where the lower half of the strut is connected with the headstock (link point). The pre-stress of both piezoelectric transducers was $T=800 \mathrm{~N}$. There was no voltage on the actuator and its electrodes were in short circuit, i.e. its behavior was totally mechanical.

The equations of motion of the system were numerically solved and the elongation of the sensor was chosen to compare the results.

First, the power spectral density of the simulated sensor elongation was examined. As expected, there is a peak at $f=330 \mathrm{~Hz}$ for the 8-DOF symbolic models as well as for the ADAMS model, as shown in Fig. 8. No other dominant frequency was observed.

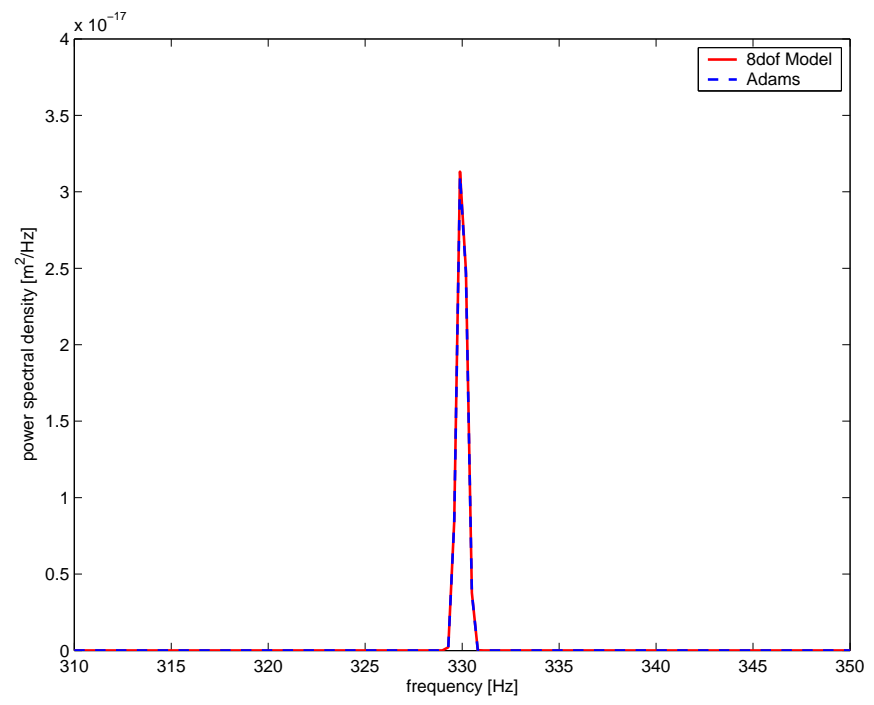

Figure 8. POWER SPECTRAL DENSITY OF SENSOR ELONGATION.

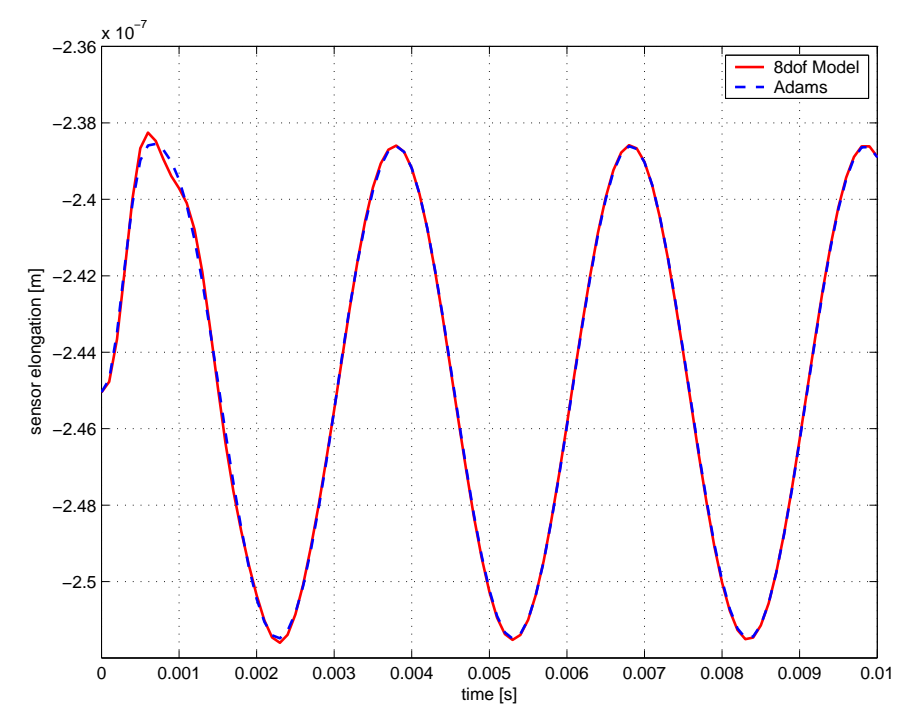

Figure 9. SENSOR ELONGATION.

The results agree with the Fourier-transformed signal shown in Fig. (5), measured on the test rig for an excitation of $f=330 \mathrm{~Hz}$ due to the vibration of the string.

Secondly, the sensor elongation itself was examined. As shown in Fig. (9), the simulation result of the 8-DOF model agree well with the simulation results of the ADAMS model, although there is a slight difference during the transient effect around $t=1 \mathrm{~ms}$. Decreasing the step size during the simulation reduced the difference during the transient effect, but the consequence was a strong increasing of the simulation time. 


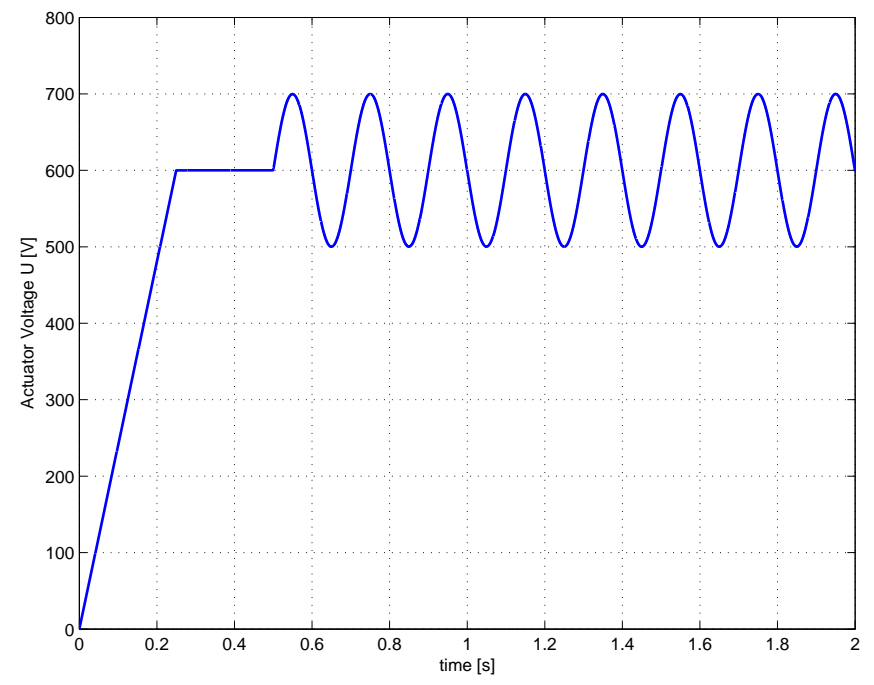

Figure 10. ACTUATOR VOLTAGE.

\section{Piezoelectric Actuator}

In a second step, the effect of a voltage applied on the piezoelectric actuator was simulatively examined. Again, the strut was vertically placed and both piezoelectric transducers were prestressed with $T=800 \mathrm{~N}$. There was no external load but the actuator voltage $U(t)$, which is shown in Fig. (10). The resulting deflection of the position of the link point on the lower half of the strut is shown in Fig. (11). Since this deflection represents an elongation of the strut, the length of the strut can be influenced via the actuator voltage. Thus, the requirement of the strut to be adjustable in length for compensating deflections due to geometric faults or process loads was successfully implemented in the symbolic model.

\section{CONCLUSIONS}

An adaptronic strut to compensate deflections of the tool center point of a machine tool due to geometric faults in the structure has been presented. A standard strut - as it is used in various industrial parallel kinematic machine tools - was split in two halves and two piezoelectric transducers were implemented as sensor element and actuator element in between the halves. The problems with measuring low-frequency signals with piezoelectric sensors were overcome by adapting the concept of a balance with vibrating string, to transform static and quasi-static signals in dynamic signals. Finally, the designed strut and its connections to the surroundings were modeled as a flexible multi-body system. Using the theory of linear graphs, the equations of motion of the system were symbolically generated applying the multi-body program DynaFlex. The equations of motion were numerically solved and the simulation results for the behavior of

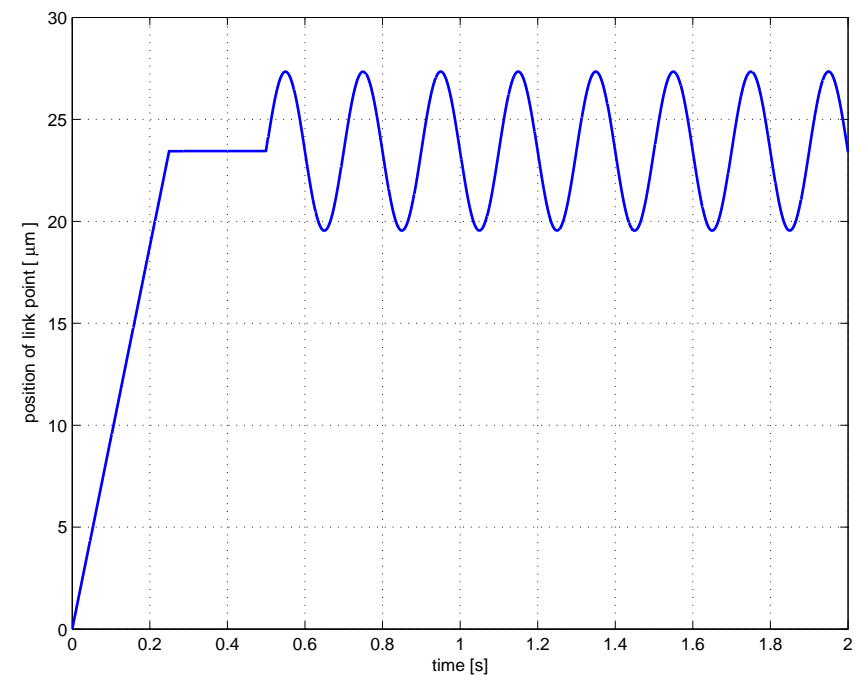

Figure 11. POSITION OF LINK POINT.

the piezoelectric transducers were presented.

The next step in the development of the adaptronic strut will be the design of a control system for compensating the influences of geometric faults. The derived equations of motion of the presented system represent a basis for a preliminary design of such a control concept. Future research will include the examination of the three dimensional motion of the adaptronic strut, the enhancement of the corresponding control system required, as well as the extension of the model to a model of a complete machine tool with parallel kinematics.

\section{ACKNOWLEDGMENT}

Financial support of this research in the frame of the Priority Programme No. 1156 "Adaptronik in Werkzeugmaschinen" by the Deutsche Forschungsgesellschaft (DFG) is gratefully acknowledged.

\section{NOMENCLATURE}

A cross-sectional area

$c_{p q} \quad$ elastic constant

$d \quad$ diameter of piezoelectric transducer

$d_{33} \quad$ piezoelectric constant

$D_{3} \quad$ electric displacement

$e_{p q} \quad$ piezoelectric constant

$E_{3} \quad$ electric field

$F \quad$ piezoelectric force

F force vector

$f_{0} \quad$ eigenfrequency of the vibrating string

$h \quad$ height of piezoelectric plates in stack transducer 


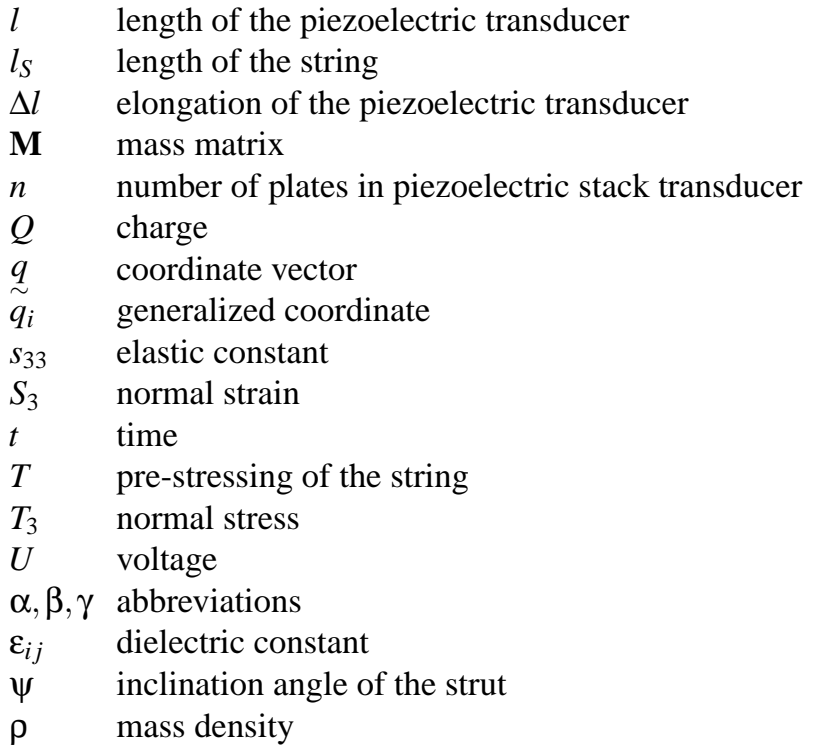

\section{REFERENCES}

[1] Hocken, J., and the Machine-Tool Task Force, 1980. "Technology of Machine Tools". Laurence Livermore Laboratory, University of California, $\mathbf{5}$.

[2] Stengele, G., 2002. "Cross Hüller Specht Xperimental, a machining center with new hybrid kinematics". The 3rd Chemnitz parallel kinematic seminar PKS, pp. 609-627.

[3] Spath, D., and Mussa, S., 2001. "Active Compensation of Machine Tool Errors with a Piezo Device”. Production Engineering, 8(2), pp. 103-106.

[4] Großmann, K., 1998. "Intelligente Funktionsmodule der Maschinentechnik". Schlussbericht zum Landesinnovationskolleg 1995 - 1998, pp. 20-114.

[5] Matsumoto, K., Hatamura, Y., and Nakao, M., 2001. "Actively controlled compliance device for machining error reduction". Annals of CIRP, 49(1), pp. 313-316.

[6] Neugebauer, R., Drossel, W.-G., Roscher, H.-J., and Schwaar, M., 2002. "Intelligent strut". The 3rd Chemnitz parallel kinematic seminar PKS, pp. 277-284.

[7] Ruschmeyer, K., 1995. Piezokeramik. expert verlag.

[8] Bill, B., 2002. Messen mit Kristallen: Grundlagen und Anwendungen der piezoelektrischen Messtechnik. Verlag Moderne Industrie.

[9] Fleischer, J., and Munzinger, C., 2005. "Aktiver Ausgleich durch Adaptronik”. Werkstatt und Betrieb(03), pp. 31-34.

[10] McPhee, J., Shi, P., and Heppler, G., 2001. "A deformation field for Euler-Bernoulli beams with application to flexible multibody dynamics". Multibody System Dynamics, 5, pp. 79-104.

[11] Shi, P., and McPhee, J., 2002. DynaFlex User's Guide. Systems Design Engineering, University of Waterloo. See also URL:

http: / / real. uwaterloo.ca/ dynaflex.

[12] Tiersten, H. F., 1969. Linear Piezoelectric Plate Vibrations. Plenum Press.

[13] von Wagner, U., 2003. Nichtlineare Effekte bei Piezokeramiken unter schwachem elektrischen Feld: Experimentelle Untersuchung und Modellbildung. GCA-Verlag.

[14] Schugt, M., 2001. "Aktor-Sensorverhalten von Piezoelementen in Kfz-Diesel-Einspritzsystemen". $\mathrm{PhD}$ thesis, Ruhr-Universität Bochum.

[15] Reiländer, U., 2003. "Das Großsignalverhalten piezoelektrischer Aktoren". PhD thesis, TU München.

[16] Andrews, G. C., 1975. Dynamics using vector-network techniques. Department of Mechanical Engineering, University of Waterloo. 